\title{
L'Équilibre de la chute : La Porte de L'Enfer et ses sources
}

\section{Boštjan Marko Turk}

\section{Synopsis}

La présente étude se donne pour but de répondre aux questions que soulève l'œuvre magistrale d'Auguste Rodin, La Porte de l'Enfer. Celle-ci puise dans les sources historiques et littéraires parmi lesquelles il faut mentionner, en premier lieu, la Divine Comédie de Dante. En réalité, en dehors des références que fournit le chantre florentin, l'œuvre d'Auguste Rodin serait impensable. Rodin lui-même avait une prédilection pour les cathédrales, les édifices qui reconstruisent le sentiment de verticalité en recompensant le porte-à-faux. Celui-ci pris dans le sens analogique fournit une explication à la structure de La Porte, surtout lorsqu'on la compare à capolavoro di Ghiberti La Porta del Paradiso qui est censé servir de modèle à Rodin. La conclusion du présent article serait que La Porte de l'Enfer est impensable en dehors du contexte philosophique du Moyen-Age.

Mots-clefs : Auguste Rodin, Lorenzo Ghiberti, Divine Comédie, enfer, verticalité, porte-à-faux, figure, Renaissance, Moyen-Age, érotisme, damnation 
La Porte de l'Enfer (Néret, 2002 : 30)1 serait l'œuvre la plus réfléchie, et pourtant la moins achevée, d'Auguste Rodin. Elle n'a été coulée dans le bronze que l'année de son décès : le sculpteur n'a jamais pu la contempler sous sa forme actuelle. Par ses origines, la sculpture remonterait à la Renaissance. C'est Lorenzo Ghiberti qui fit la Porta del Paradiso (Ghiberti, 2005 : 70) : celle-ci aurait servi de modèle à Rodin. La dissemblance entre les deux paraît pourtant frappante : "Originally conceived as an 'infernal' response to Ghiberti's structured and orderly 'Gates of Paradise' Rodin's gates are all disorder and chaos " (Néret, $2002: 26$ ).

La disparité s'expliquerait par le fait que les éléments phénoménologiques (structurels) dont se sont servis l'un et l'autre pour exécuter le capolavoro sont différents. La critique a souvent mis l'accent sur le fait que Les Portes de l'Enfer doivent la partie essentielle de leur inspiration à la Renaissance : "Toutefois, c'est surtout à la Renaissance que l'cuvre fait référence, avec sa composition en lignes orthogonales, ses pilastres ornés et son entablement à modillons $»{ }^{2}$ Or, Rodin avait une prédilection pour la période gothique ${ }^{3}$ qu'il considérait comme "l'bistoire de la France " et comme "l'arbre de toutes nos généalogies". Étant donné que le sculpteur était en même temps un spéculatif, la citation suivante ne devrait pas surprendre :

"Avant de disparaître moi-même je veux du moins avoir dit mon admiration pour ces merveilles, moi qui ai le bonheur de les aimer et qui ai goûtées devant elles les plus belles jouissances de ma vie. Je veux célébrer ces pierres si tendrement amenées à la beauté par d'humbles et savants artistes, ces moulures amoureusement modelées comme des lèvres de femme, ces séjours de belles ombres, où la douceur sommeille dans la force, ces nervures fines et puissantes qui jaillissent vers la voûte et s'inclinent sur l'intersection d'une fleur, ces rosaces des vitraux dont l'appareil est pris au soleil couchant et au soleil du matin» (Morel, $2011: 104)$.

Deux constatations s'ensuivent : la prépondérance ontologique sur laquelle reposent les principes architécturaux de ces édifices (la pierre "amenée à la beauté", étant donné la finalité de cette opération) et le style gothique, ${ }^{4}$ comme l'analogie de la création de Rodin.

La dernière paraît être d'une importance majeure :

$1 \quad$ Nous ne donnons dans la suite du texte plus cette référence: elle serait superflue et redondante. Au lieu de cela on se sert de l'abréviation où l'on ne retient que le premier élément du syntagme: La Porte.

$2 \mathrm{http} / /$ www.musee-rodin.fr/fr/collections/sculptures/la-porte-de-lenfer-troisieme-maquette, consulté le 30 juin 2016

3 Pour le style ogival en France comparer: Aubert, Marcel : Cathédrales et Trésors gothiques de France. Paris : Arthaud, 1958.

4 A comparer: Pobé, Marcel : Splendeur gotbique en France. Paris : Branin 1960 et Réau, Louis : L'Art gothique en France. Paris : Le Prat 1954. 
"Tout le monde sait que le corps humain, dans le mouvement, porte à faux. L'équilibre est rétabli par des compensations. La jambe qui porte, rentrant sous le corps, sert de pivot au poids entier et fait seule, en cet instant, l'unique et total effort. La jambe qui ne porte pas sert seulement à modeler, à moduler les degrés de la station et la modifie, soit insensiblement, soit rapidement, jusqu'à se substituer à la jambe qui porte et à la libérer. C'est ce que le peuple appelle 'se défatiguer' en portant d'une jambe sur l'autre le poids du corps : ainsi une cariatide qui changerait d'épaule son fardeau [...]. Les cathédrales sont ce porte-à-faux compensé, instinctivement employés par la vie qui ont inspiré les oppositions et les équilibres gothiques »(Morel, 2011 : 107).

Une lecture approfondie du texte sur Rodin, l'essai de Rainer Maria Rilke, dévoile le caractère pertinent du " porte-à-faux compensé " 5 ayant pour conséquence "la naissance du geste » (Rilke, 2009 : 28). Au geste qui est la séquence évolutive de cette première démarche, Rilke identifie le soubassement de l'acte créateur de la sculpture entière : Le geste qui est le mouvement devient la souche d'élection de cet art : "On pourrait dire de ce geste qu'il repose comme enfermé dans un bourgeon dur : il s'ouvre et voici qu'il surgit Saint Jean ». ${ }^{6}$ Celui-ci est le premier à incarner l'équilibre désormais inébranlable.

"Ce Saint Jean est le premier homme qui marche dans l'ouvre de Rodin. [...] Il marche comme si toutes les étendues du monde étaient en lui et comme s'il les distribuait de son pas. Il marche. Ses bras témoignent de cette marche et ses doigts sécartent et semblent dans l'air faire signe de la marche [...]. Beaucoup d'autres viennent derrière lui. Viennent les Bourgeois de Calais et chacun de leurs pas semble préparer le grand pas provoquant de Balzac» (Rilke, 2009 : 29).

Le mode de locomotion incarnant le premier geste est le phénomène le plus naturel de l'homme. Il ne prend son sens que juxtaposé avec l'autre prodige qui lui donne son sens définitif, celui de la spiritualité.

"J'étudiai ensuite de la même façon le Saint Jean-Baptiste. Et je vis que le rythme de cette figure se ramenait encore, comme me l'avait dit Rodin, à une sorte de l'évolution entre deux équilibres. Le personnage, appuyé d'abord sur le pied gauche qui pousse sur le sol de toute sa force, semble se balancer à la mesure que le regard se porte vers la droite. [...] Or la science du sculpteur a consisté précisément à imposer au spectateur toutes ces constatations dans l'ordre où je viens de les indiquer, de manière que leur succession donnât l'impression du mouvement. Au surplus, le geste du Saint Jean-Baptiste recèle de même que celui de l'Age d'airain une signification spirituelle. Le prophète se déplace avec une solennité presque automatique. On croirait entendre ses pas sonner comme ceux de la statue du Commandeur. [...] Ainsi la marche, ce

5 Voir supra.

6 Ibidem. 
mouvement, si banal d'ordinaire, devient ici grandiose parce quelle est l'accomplissement de la mission divine » (Gsell, $1911: 49)$.

Dans la démarche des figures de l'univers de Rodin, il y a donc le même porteà-faux compensé que dans les édifices hiératiques. L'équilibre qui leur permet d'être, de compenser la chute est précaire de nature, et pourtant grandiose.

Il doit constituer le trait essentiel - au moins en ce qui concerne ces structures qui se référent volontairement au contexte religieux. Et La Porte de l'Enfer relève exclusivement de ce domaine. En le comparant à la Porta del Paradiso (Ghiberti, 2005 : 70), il y a une dissemblance qu'on remarque en premier lieu. Lorenzo Ghiberti a divisé l'espace de deux baies en dix panneaux, tandis que chez Rodin une telle répartition n'a pas été faite. Le Florentin a ainsi, volontairement ou non, oblitéré toute impression de profondeur, de l'aplomb. Il a rendu son œuvre orthogonale pour suspendre toute tendance, surtout celle de la verticalité. Rodin travaillait dans le sens inverse. Au centre de la composition, il a instauré un trumeau sur lequel - au milieu du tympan - est assise sur un chapiteau la figure principale, $L e$ penseur, qui regarde strictement en bas. Il est surplombé par la composition Trois ombres, dont les mains pointent aussi vers le fond. Rilke à ce propos constate :

"Le penseur est au centre de la porte, bien que, au-dessus de lui encore, à la hauteur du cadre, trois hommes soient debout. La profondeur agit sur eux et les tire de loin. Ils ont rapproché leurs têtes, leurs trois bras sont tendus en avant, ils courent ensemble et désignent le même point, en bas, dans l'abìme qui les attire de toute sa lourdeur " (Rilke, 2009 : 41).

Lespace des deux panneaux est rempli de figures plastiques qui sont toutes distordues lors de leur chute sans fin. L'impression qui se dégage de l'ensemble est le vertige de la verticalité intégré à la chute, corporelle et spirituelle à la fois, sans aucune possibilité de porte-à-faux contrebalancé.

Or, la verticalité, impliquant un équilibre restauré, est le sens des édifices gothiques :

"Cet élan en hauteur de tous les organes de l'édifice, ces masses qui fusent de toutes parts vers le ciel, ce hérissement de tours, de flèches, de clochetons, de pinacle, c'est bien ainsi qu'il faut se représenter comment Notre-Dame de Laon ou Notre-Dame de Reims ont été conçues à l'origine» (Lambert, 1943 : 7).

La verticalité qui tend vers la hauteur est le contraire de la chute. La Divine Comédie (Dante, 1910) est structurée d'après le même principe. Où chercher les raisons de l'effondrement ? L'enfer de Dante et celui de Rodin sont prismatiques, spectraux. L'enfer est la perte la plus immédiate de l'équilibre, le porte-à-faux 
jamais neutralisé. Sur chaque élément, si minime qu'il soit, pèse le poids de l'ensemble. Les effets que la lourdeur de l'enfer fait ressentir aux protagonistes de la Divine Comédie sont saisis jusqu'aux détails les plus spectaculaires. Les tensions se transmettent verticalement. Le comte Ugolin della Gherardesca placé au dernier cercle et intégré au centre du relief de La Porte, ronge le crâne du prélat Ruggeri Ubaldini. C'est un geste prélevé dès le moment de la naissance qu'on laisse durer pour l'éternité. Ugolin fut un des tyrans les plus maléfiques qui vécurent dans l'Italie du 13è siècle. Il a livré sa ville natale, Pise, aux mains des ennemis ce qui ne l'a ensuite pas empêché de s'accaparer du pouvoir en se servant des manœuvres corrompus. Comme le cannibale fait partie de la tribu de ceux qui ont trahi leur patrie ou leurs compagnons, ce mouvement du corps est à jamais figé dans l'eau congelée. Il s'agit en même temps d'une communauté plus vaste régie par les lois morales. Le compte a commis une action néfaste vers l'ensemble structurel que l'homme concevait comme reflet de la volonté suprême. Ugolin appartient au groupe de ceux qui ont été le plus déséquilibrés se trouvant sur le lieu des supplices les plus infâmes. Il paraît que l'univers entier pèse sur sa culpabilité. Du haut de La Porte c'est Adam qui pointe vers le bas, aggravant sa peine. Ugolin et ses fils sont devenus l'analogie de la douleur, étant séparés pour l'éternité de la finalité de l'univers médiéval ainsi que de son ordre prescrit. C'est pour cette raison qu'ils figurent au milieu du relief central de La Porte.

La Divine comédie (Dante, 1910) est l'œuvre littéraire en même temps que le paysage spirituel. Par son pouvoir analogique il correspond bien à la sculpture en trois dimensions où se reflètent tous les actes que l'être humain accomplit sous les auspices de l'éternité. Chaque geste du mouvement y compte.

Les éléments qui caractérisent l'espace de La Porte sont les suivants. D'abord il y a l'impression omniprésente de l'horreur figée dans la verticale. C'est la perspective de la verticalité qui implique la chute irréversible vers le bas, conséquence logique de la métaphysique morale du Moyen-Âge. C'est le trait principal de $L a$ Porte. Qu'on rappelle que l'œuvre exhibe la synthèse des composants antérieurs qui figuraient déjà auparavant comme entités autonomes.

Dans La Porte ils sont intégrés dans l'ultime synthèse qui indique l'irréversibilité sans limite. ${ }^{7}$ Lespace de l'horreur que renferme la porte est vertical et horizontal à la fois. Il y a la peur de la chute ainsi que l'appréhension de ce qui pourrait être derrière. Les deux dimensions agissent simultanément sur la conscience de l'homme. L'instrument, avec lequel la nature l'a muni pour contacter l'espace fermé du côté opposé à la face, est la main. La Grande Main crispée (Goldscheider, 1962 : 81) se lève afin de saluer : elle ouvre et referme la porte donnant l'illusion de pouvoir ralentir le cours des événements. En même temps son spasme traduit

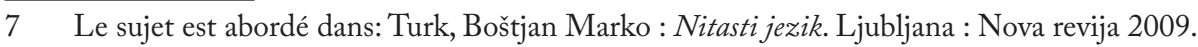


la douleur ineffable de l'adieu. Il paraît que la contraction qui l'a figée ne relâchera jamais. L'image de La Grande Main crispée pourrait être parallèle à celle du Désespoir (Matiussi, 2012 : 100) qui est, elle aussi, fondée sur le resserrement sans remède d'un corps capturé à jamais.

Ce n'est pas toujours le spasme qui contracte les muscles d'un corps jusqu'à l'infini. Le contraire peut bien mener à une situation pareille. Qu'on se rappelle l'engourdissement qui s'empare du fils de Dédale lors qu'il perd la poussée verticale est se trouve précipité dans la mer. Rodin a rebaptisé le mythe et s'en est servi à plusieurs reprises. Il y a L'Illusion, Fille d'Icare (Mattiussi, 2012 : 32) puis Le Martyre (Néret, 2002 : 33) ainsi que maintes préexploitations du même sujet mais toujours en fonction de l'affaissement ou de la descente : "L'Illusion, la Fille d'Icare, cette éblouissante transformation en une chose d'une longe chute désarmée «(Rilke, 2009: 43).

Le spasme qui passe à l'engourdissement fait songer soit à la léthargie de laquelle il n'y a plus de réveil, soit à un songe bizarre dont le contenu s'évanouira à jamais. Il laisse entrevoir la naissance de la conscience en face de la damnation. Dans la proximité immédiate d'une telle inspiration gémit l'image de La Jeune Parque (Valéry, 1957 : 96) dont la conscience s'éveille à la vie tandis que celle des figures de Rodin glisse vers la mort. On pourrait y joindre La Danaïde (Mattiussi, 2012 : 67) telle qu'elle a été saisie dans l'appréhension de Rainer Maria Rilke :

"Peut-être est-ce à cette époque qu'a vu jour cette Danaïde qui, hors de ses genoux, s'est jetée dans sa chevelure liquide. On éprouve une impression merveilleuse à faire seulement le tour de ce marbre: le long, le très long chemin autour de la courbe de ce dos, richement déployée, vers le visage qui se perd dans la pierre comme dans un grand sanglot, vers la main qui, pareille à une dernière fleur, parle encore une fois doucement de la vie, au coeur de la glace éternelle du bloc »(Rilke, 2009 : 42).

Étant donné que la courbe est l'analogie d'un songe qui invite à l'au-delà les corps dans les formes arrondies ne font qu'un avec l'entité qui leur est préposée. $\mathrm{Au}$ sommet de La Porte il y a la sculpture Les Trois Ombres. Celle-ci détermine l'ensemble. Les mains de trois personnages pointent vers le bas. A gauche il y a le premier des protoparentes, Adam. Formant le tout avec deux statues anonymes, il laisse pressentir l'horreur derrière l'espace vide. Rodin avait l'intention de munir La Porte d'une gravure contenant les vers de la Divine Comédie: "Vous qui entrez, laissez toute espérance » (Dante, 1910 : 12). Finalement il s'est ravisé, non sans raison. Le chemin par où l'on passe dans la cité des pleurs et à l'éternelle douleur que subit la race perdue semble être mieux indiqué par la perspective que dénotent les membres des trois figures. Il paraît que dans l'espace au fond, en arrière, il y a l'accumulation d'une énergie dévastatrice qui menace de briser toute créature. 
Comme si la diachronie, le mouvement que trace la ligne de l'espace-temps de la condition humaine changerait en synchronie fournissant à l'entourage immédiat de La Porte un vide ayant pour fonction de créer un univers nouveau, négatif cette fois. Cet univers serait doté d'une force inégalable menaçant de faire disparaitre tout ce qui est devant lui. C'est l'analogie efficace de la damnation éternelle.

En fait, l'espace vide derrière Les Trois Ombres laisse présager l'ineffable analogie de l'anathème. Le vide suffit. Il n'est pas besoin d'évoquer les "pals et les grils » (Sartre, $1947: 12$ ) les figures que le souffle vient de quitter, répondent seules à ce genre d'exigences. L'Esclave de Michel-Ange (Arbour, 1962 : 119) apparenté à ce groupe permet de comprendre pourquoi Rodin prit cette statue pour modèle : le motif en était qu'il avait besoin d'une opposition. ${ }^{8}$ La plénitude d'un corps humain tel que la Renaissance l'a inspiré permet la comparaison avec les trois figures de La Porte. Bien que les esclaves de Michel-Ange fassent proprement partie de l'art funéraire, leur auteur ne manquait pas d'insister sur les éléments qui représentaient une force devant laquelle tout reculait, même la mort (et le péché comme sa partie intégrante) : "Le tombeau de Jules II est conçu, à la Renaissance, comme un édifice consacré au culte de la personnalité, qui réunit dans sa composition devenue monumentale, architecturale même, les symboles de la vie terrestre du défunt et de son accession au domaine des élus " (Arbour, 1962 : 118). Dans ce sens, même un esclave intégré au tombeau est un symbole de la victoire définitive : «Les Esclaves et les Victoires dont il faut chercher les origines dans l'antiquité païenne, symboliseraient la victoire de l'Eglise et de la chrétienté sur l'impiété et l'incroyance " (Arbour, 1962 : 121). Par contre, si l'on vidait ces figures de l'élan vital qui les redresse vers le haut, elles s'affaisseraient, car elles auraient perdu leur principe primordial. Elles deviendraient l'une des trois entités de la composition exposée à l'Hôtel Biron. Au lieu de tendre vers le haut, elles retomberaient vers le bas. Rodin avait besoin d'un modèle qui mettrait en relief l'opposition irréductible. C'est pour cette raison qu'il s'est tourné vers le Florentin.

L'appréhension que le palimpseste de Michel-Ange laisse derrière Les Trois Ombres et La Porte en tant qu'ensemble permet de contraster la tension verticale avec celle de l'horizontalité telle quelle se produit par l'impression de la porte fermée et l'épouvante de ce qui pourrait être de l'autre côté. Entre la pulsation spasmodique et le relâchement intégral il y a la dimension à travers laquelle s'exprime la vie. C'est là l'espace de sa lignée vitale. Entre l'intérieur de la contraction mortelle et la rêverie profonde il y a la place de la sexualité. Celle-ci est chez Dante et chez Rodin analogique : elle est pars pro toto dénotant la semence de la vie, sa continuité.

8 Rodin fut sensible dès le début à la représentation graphique par laquelle le sculpteur Florentin captivait les formes des phénomènes de la pensée: "Il passe beaucoup de temps à la bibliothèque Sainte-Geneviève, inaugurée quatre ans plus tôt. En consultant par hasard des livres de gravures d'après Michel-Ange, c'est la révélation: il décide qu'il fera carrière dans le dessin «(Pinet, 1998:14) 
Qu'on prenne la compagne du premier homme, Eve (Goldscheider, 1962 : 61), une des créations du sculpteur les plus réussies. Son corps laisse perdurer le souvenir du péché. Les mains qui couvrent le visage indiquent la honte. Comme si elle était à nu ne pouvant se réfugier nulle part devant les flèches qui l'attaquent. Son corps est lâche et flou. C'est le corps détendu de quelqu'un qui est épuisé et rassasié de l'amour charnel. Son pèlerinage touche à son terme. Les cercles que dessine la grossesse autour du ventre laissent entrevoir la conséquence fatale de l'évènement fatidique. Eve est enceinte : la progéniture qui naîtra sera maudite : la damnation s'en est emparée avant même qu'elle ait eu le temps de s'y soustraire. Eve, c'est la patrie de la mort, c'est pour cette raison que Rodin l'a figurée comme un post festum sui generis. Elle a détourné le visage de Dieu étant séparée à jamais de l'énergie qui la comblait, il y a un moment. Désormais le dénominateur commun entre elle et son compagnon sera le manque de l'élan vital : ils sont comme une outre jadis remplie qui s'est vidée pour de bon.

La sexualité est la force puissante des rythmes de la vie. Suivant cette idée on arrive au paroxysme du spasme voluptueux qu'expriment les figures de Rodin. Le plaisir sensuel est une dérivation morphologique de l'enfer, un porte-à-faux mal compensé. La porte, c'est de la perte. Notamment, il y a deux personnes unies, intégrées à $L a$ Porte qui soulignent la prédilection du sculpteur pour les enjeux du plaisir érotique : "Les couples mettant en scène l'exubérance de leurs ébats amoureux furent l'un des thèmes privilégiés de Rodin» (Mattiussi, 2013 : 77). Une production plastique est mémorable dans ce sens-là. C'est Paolo et Francesca (Rodin, 2014 : 26), une analogie de l'amour aveugle et non accompli. Il semble que "la fuite de leur chute entraîne les étoiles»(Rilke, 2009 : 42). Dante partagea leur fureur jusqu'aux bout de ses forces : "Pendant qu'ainsi parlait l'un des esprits, l'autre pleurait tellement que de pitié je défaillis, comme si je me mourais; et je tombai comme tombe un corps mort». (Dante, 1910 : 22). En s'appuyant sur le commentaire suivant, on peut constater la même chose : "Dans l'Enfer il n'y a presque pas de figure qui fût davantage plus chère à son auteur qu'Ulysse celui qui appelle à la vertu de la connaissance. La seule qui puisse le rivaliser est Francesca, l'icône de l'amour» (Capuder, 2005 : 228). Rodin est identique. À côté du Penseur (Néret, 2002 : 34), apparenté aux figures cruciales de La Porte, il y a Le Baiser (Néret, 2002 : couverture). Il a été conçu afin de figurer dans La Porte mais l'auteur ne put jusqu'à la fin se décider à l'inclure dans l'ensemble. La sculpture trace l'instant intense du plaisir sensuel de Paolo et Francesca : ils se livrent sans aucune défiance à l'émotion violente comme ils s'y livraient lorsque Giovanni Malatesta les surprit, transformant par un poignard le moment du bonheur dont ils jouissaient pour l'éternité. Ils fonctionnent désormais comme un couple doté d'un équilibre difficile, se reconstituant d'une circonstance à l'autre, trébuchant en conséquence d'un porte-à-faux mal balancé. "L'anatomie de Rodin n'obéit pas à la loi immuable de chaque corps humain pris individuellement, 
elle est l'éphémère configuration d'un instant » (Mattiussi, 2013 : 45). Suivant cette perspective on retrouve dans $L a$ Porte plusieurs représentations sublimes de leur tragique amour. Celui-ci devint par la suite l'emblème de la contenance générale de l'homme quant à ce sentiment. Fugit Amor (Néret, 2002 : 46) exhibe les deux amants comment ils se déversaient l'un dans l'autre lorsqu'ils traversaient le temps. Comme si Paolo voulait se soustraire à l'étreinte de sa belle compagne afin de devenir par la suite l'Enfant prodigue (Goldscheider, 1962 : 69) qui - les mains grandes ouvertes - plaint la patrie, le bonheur et l'amour perdus qu'il ne ressentira plus. Le couple représenté par Le Baiser est réuni en ce qui conférerait à ce sentiment son but ultime. Rodin se focalise sur leur étreinte, notamment sur la passion qui rend les deux figures désormais inséparables. Elles sont tordues par la concupiscence qui fait fusionner leurs corps. L'emblème de leur posture est de nouveau la main, celle de Paolo avec laquelle il déborde sur la hanche de Francesca. On assiste à une contradiction ontologique lorsque l'énergie vitale se concentre sur la possession de l'autre étant en même temps aiguillée vers le point cardinal de la séparation, da la fin. Comme si l'étendue avoisinante de la statue était l'interstice où tout serait condamné à s'avancer vers son déclin perdant sa cohésion interne. Pourtant, Paolo et Francesca sont une des mises en abyme les plus réussies de l'équilibre qui est à la fois l'équilibre incontournable de l'existence : néanmoins, son porte-à-faux ne pourrait à aucun moment être compensé. Ainsi, les deux amants révèlent de manière pars pro toto l'énigme imparable de l'être humain. C'est l'érotisme intégral qui s'affirme par le biais de l'antinomie eros - thanatos conférant à la verticalité de La Porte le sens complet. Notamment, le propre de l'amour, c'est d'être d'avance décidé comme cette force qui précipite inéluctablement vers le fond.

La réflexion critique du chef-d'œuvre de Rodin ne peut donc pas se passer d'envisager la longue méditation poursuivie tout le long de sa carrière au sujet des édifices gothiques et de leurs éléments structurels, physiques et métaphysiques. Les cathédrales sont bâties sur le spirituel elles ont été conçues de façon à refléter la tension perpendiculaire à l'horizon suivant la direction de la pesanteur. Celleci a une dénotation morale implicite. Il y va de même pour la Divine Comédie (Dante, 1910). L'Enfer y est mis au point grâce à la forme d'un vortex creux qui, à travers les neufs cercles, disparaît dans la réprobation.

La Porta del Paradiso de Ghiberti a été construite dans la perspective opposée. Contre le dynamisme vertical de l'âge gothique s'y dresse l'équilibre stable, conséquence naturelle d'une période qui avait confiance en l'être humain. C'est la Renaissance. Il s'agit d'un univers dont l'homme est le seul maître. De son côté, l'univers du Moyen-Âge dans le sens général du terme se pliait aux lois contraires. Loin d'être une autorité indisputable, l'homme est plongé dans un environnement opaque où même ses sentiments les plus inhérents (l'érotisme en tant que la quête de l'autre) paraissent contribuer à sa perte. 
Toutefois Auguste Rodin considérait que le dynamisme des lignes verticales tel qu'expriment la croyance et habitudes intellectuelles qui régissaient la collectivité du temps médiéval était la source de l'inspiration bien plus inépuisable que ne sont la philosophie et l'anthropologie de la Renaissance. C'est au même instant la seule clé qui puisse mener derrière les arcanes de La Porte.

\section{BIBLIOGRAPHIE}

Aligheri, Dante : la Divine Comédie (trad. Robert de Lammenais). Paris: Flammarion, 1910.

Arbour, Renée : Michel-Ange. Paris : Somogy, 1962.

Aubert, Marcel : Cathédrales et Trésors gothiques de France. Paris : Arthaud, 1958.

Capuder, Andrej : «Komentarji ». Dante : Pekel, Celje : Mohorjeva, 2005.

Ghiberti, Lorenzo: Porta del Paradiso. Roma : G.E.P., 2005.

Goldscheider, Cécile : Rodin. Paris : S.P.A.D.E.M, 1962.

Gsell, Paul : «Le mouvement dans l'art ». Auguste Rodin - l'art. Paris : Grasset, 1911.

Lambert, Elie : Le Style gotbique. Paris : Larousse, 1943.

Mattiussi, Véronique : Rodin. Paris : Seuil, 2012.

Musée Rodin : Portal. Http://www.musee-rodin.fr/fr/collections/sculptures/laporte-de-lenfer-troisieme-maquette, consulté le 30 juin 2016.

Néret, Gilles : Rodin. Cologne : Taschen, 2002.

Pinet, Hélène : Rodin - Les Mains du génie. Paris : Gallimard, 1998.

Pobé, Marcel : Splendeur gothique en France. Paris : Branin, 1960.

Réau, Louis : L'Art gothique en France. Paris : Le Prat, 1954.

Rilke, Rainer Maria : Sur Rodin. Bruxelles : André Versaille, 2009.

Rodin, Auguste : "Le Soir des cathédrales ». Jean-Paul Morel : Faire avec ses mains ce quion voit. Paris : Mille et une nuits, 2011.

Rodin, Auguste : Euvre. Lyon : Fage, 2014.

Rodin, Auguste : «Pour faire aimer les cathédrales ». Jean-Paul Morel : Faire avec ses mains ce quion voit. Paris : Mille et une nuits, 2011.

Sartre, Jean-Paul : Huis clos. Paris : Gallimard, 1947.

Turk, Boštjan Marko : Nitasti jezik. Ljubljana : Nova revija, 2009.

Valéry, Paul : Euvres complètes, Paris : Gallimard, 1957. 
Boštjan Marko Turk

Université de Ljubljana Bostjan-marko.turk@guest.arnes.si

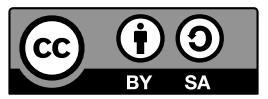

\section{Porušeno ravnovesje: Avgust Rodin in filozofske matrike Vrat pekla}

Pričujoči članek razdeluje literarno in filozofsko tematiko, ki je navdahnila eno največjih del Augusta Rodina, njegova Vrata pekla. Osnova je Dantejeva Božanska komedija, veliko pa delo dolguje tudi Rodinovemu premišljevanju gotskih katedral, predvsem njihovemu temeljnemu občutju, to je vertikalnosti. Prav slednja daja pogubljenim figuram Vrat pekla zagon nepovratnega drsenja proti dnu. V tem se Rodinova plastika bistveno ločuje od Vrat nebes renesančnega avtorja Lorenza Ghibertija, četudi jih je umetnostna zgodovina vselej jemala kot model, po katerem naj bi Rodin oblikoval Vrata pekla. Vendar se ena in druga umetnina v filozofskem sporočilu in arhitekturnem konceptu toliko razlikujeta, da je nekaj takega tudi teoretično nemogoče.

V Vratih pekla Rodin poustvarja dinamiko človekovega življenskega erosa v luči večnosti, smrti in pogubljenja: to so srednjeveške kategorije mišljenja, brez katerih bi Vrata ne bila takšna kot so. S tem pa smo stopili daleč stran od renesančne inspiracije, v ospredju katere je stal optimističen pogled na človeka, kot središče sveta.

Ključne besede: Auguste Rodin, Lorenzo Ghiberti, Božanska komedija, pekel, vertikalnost, kipi, drža v neravnovesju, renesansa, srednji vek, erotika, pogubljenje.

\section{The Broken Balance: Auguste Rodin and Philosophical Matrix of La Porte de l'Enfer}

The study tries to answer the questions opened up by the work of Auguste Rodin, La Porte de l'Enfer. It was inspired by earlier historical and literary sources among which Dante's Divine Comedy has to mentioned first, as it cannot be imagined without it. The study compares the structure of La Porte de l'Enfer with Ghiberti's masterpiece La Porta del Paradiso. The study concludes that the discussed Rodin's work is impossible to comprehend outside of the medieval philosophical context.

Keywords: Auguste Rodin, renaissance, Lorenzo Ghiberti, Divine Comedy 